\title{
The SPA arrangement of the branches of the upper trunk of the brachial plexus: a correction of a longstanding misconception and a new diagram of the brachial plexus
}

\author{
Amgad Hanna, MD \\ Department of Neurological Surgery, University of Wisconsin, Madison, Wisconsin
}

OBJECTIVE Brachial plexus (BP) diagrams in most textbooks and papers represent the branches and divisions of the upper trunk (UT) in the following sequence from cranial to caudal: suprascapular nerve, anterior division, and then posterior division. This concept contradicts what is seen in the operating room and is noticed by most peripheral nerve surgeons. This cadaveric study was conducted to look specifically at the exact pattern of branching of the upper trunk of the BP.

METHODS Ten cadavers (20 BPs) were dissected. Both supra- and infraclavicular exposures were performed. The clavicle was retracted or resected to identify the divisions of the BP. A posterior approach was used in 2 cases.

RESULTS In all dissections the origin of the posterior division was in a more cranial and dorsal plane in relation to the anterior division. In most dissections the supra scapular nerve branched off distally from the UT, giving it the appearance of a trifurcation, taking off just cranial and dorsal to the posterior division. The branching pattern of the UT consistently had the following sequential arrangement from cranial and posterior to caudal and anterior: suprascapular nerve (S), posterior division $(P)$, and anterior division $(A)$, hence the acronym SPA.

CONCLUSIONS Supraclavicular exposure of the BP exposes only the trunks and divisions. Recognizing the "SPA" arrangement of the branches helps in identifying the correct targets for neurotization, especially given that these 3 branches are the most common targets for BP repair. Understanding the anatomy means better surgical planning and better patient outcomes.

http://thejns.org/doi/abs/10.3171/2015.5.JNS15367

KEY WORDS anatomy; brachial plexus; divisions; suprascapular nerve; upper trunk; peripheral nerve

\section{$\mathrm{W}$} E have all been taught to draw the brachial plexus (BP) with the anterior division (A) of the upper trunk (UT) more cranial than the posterior division (P)., $4,5,7,14-16,18$ For the peripheral nerve surgeon familiar with BP reconstruction, this is anatomically incorrect; the correct arrangement should be $\mathrm{P}$ cranial to A. Dealing with BP trauma is already challenging because of scar tissue that sometimes masks the normal anatomy. Without the knowledge of normal anatomical concepts, one may be grafting into the wrong targets. In an effort to better understand the relationship of the divisions of the UT, 20 BPs were dissected in embalmed cadavers, looking specifically at the branching pattern of the UT.

\section{Methods}

Ten embalmed cadavers were used for this project.
Both sides (20 BPs) were dissected, mostly via an anterior approach. A skin incision was made along the posterior border of the sternocleidomastoid (SCM) muscle or horizontally. The platysma was opened. The omohyoid, suprascapular, and transverse cervical vessels were transected. The supraclavicular pad of fat was reflected laterally from the posterior border of SCM. The trunks of the BP were then identified between the anterior and middle scalenes. Special attention was devoted to identifying the branches and divisions of the UT. They were also followed proximally to their origin from the C-5 and C- 6 spinal nerves.

Infraclavicular exposure was achieved through the deltopectoral groove. The pectoralis minor was transected, which allowed exposure of the cords of the BP and the axillary artery. For full exposure of the divisions, the clavicle was either retracted or transected.

ABBREVIATIONS $\mathrm{A}=$ anterior division; $\mathrm{BP}=$ brachial plexus; $\mathrm{LT}=$ lower trunk; $\mathrm{MT}=$ middle trunk; $\mathrm{P}=$ posterior division; $\mathrm{S}=$ suprascapular nerve; $\mathrm{SCM}=$ sternocleidomastoid; UT = upper trunk.

SUBMITTED February 14, 2015. ACCEPTED May 14, 2015.

INCLUDE WHEN CITING Published online November 27, 2015; DOI: 10.3171/2015.5.JNS15367. 
Posterior exposure of the $\mathrm{BP}$ was carried out twice to facilitate complete illustration. A posterior paramedian incision was made between the midline and the medial border of the scapula. The trapezius, rhomboids, and levator scapulae were transected. The serratus posterior superior, scalenus posterior and medius, and the first rib were resected, thus exposing the posterior aspect of the BP. The long thoracic nerve was first encountered and retracted laterally to expose the trunks and divisions.

\section{Results}

\section{Anterior Approach}

The findings from the anterior approach were consistent across all dissected specimens. Despite some variability in the level of origin of the suprascapular nerve from the UT, the order was the same from cranial and dorsal to caudal and ventral: suprascapular nerve (S), posterior division $(\mathrm{P})$, and anterior division $(\mathrm{A})$, respectively (20 of 20, 100\%) (Fig. 1). In most specimens, this appeared as a trifurcation (14 of 20,70\%). Occasionally, S was a few millimeters proximal to the bifurcation of the UT ( 1 of 20 , $5 \%$ ). More frequently, the take-off of A was more proximal to $\mathrm{P}$ and $\mathrm{S}$, which made $\mathrm{S}$ appear as a branch of $\mathrm{P}(5$ of 20,25\%) (Fig. 2). The UT was formed by the junction of C-5 and C-6, and occasionally C-4, spinal nerves. In most specimens, we were able to find the nerve to subclavius. The middle trunk (MT) (C-7) was in a plane caudal and dorsal to the UT, and the lower trunk (LT) (C8-T1) was in a plane caudal and dorsal to the MT. The subclavian artery was observed anterior to the LT. The anterior divisions of the UT and MT joined to form the lateral cord, while the anterior division of the LT continued as the medial cord. The posterior divisions of the 3 trunks met to form the posterior cord. Data were available from 16 BPs. Most of the time, the posterior divisions of the UT and MT met first, and were then joined more distally by the posterior division of the LT ( 8 of 16, 50\%), and in 1 of these specimens this was after the take-off of the axillary nerve. Less often the posterior divisions of the MT and LT met first, then joined the UT ( 6 of 16, 37.5\%), and in 1 of these specimens this was after the take-off of the axillary nerve. The exception was that the 3 posterior divisions met at 1 point to make up the posterior cord (1 of $16,6.25 \%)$. In another specimen $(6.25 \%)$ there were 2 posterior divisions to the MT, a cranial one that joined the posterior division of the UT, and a caudal one that joined the posterior division of the LT. These ultimately met to form the posterior cord (Fig. 3).

\section{Posterior Approach}

From the posterior approach, once the long thoracic nerve was retracted laterally, the UT trifurcation was consistent with the findings from the anterior approach, namely from cranial and dorsal to caudal and ventral: S, P, and A, respectively (Fig. 4) (2 of 2, 100\%).

\section{Discussion}

There has been significant confusion in the literature in regards to the divisions of the upper trunk of the BP. Part of the confusion is caused by how we draw and teach the anatomy of the BP. It may be due to ease or convenience that we always draw or represent the BP with the anterior division of the UT more cranial than the posterior division. ${ }^{4,5,7,9,14-17}$ This probably dates to the time of Vesalius, in his illustration of the BP in $1555^{18}$ (Fig. 5). We may have just been perpetuating a myth. Most of the peripheral nerve surgeons know from experience that the correct arrangement from cranial and dorsal to caudal and ventral is $\mathrm{S}, \mathrm{P}$, and A, sequentially. Understanding the correct anatomy is extremely important when dealing with trauma cases, especially if only a supraclavicular exposure is performed. An incorrect concept may result in reinnervating

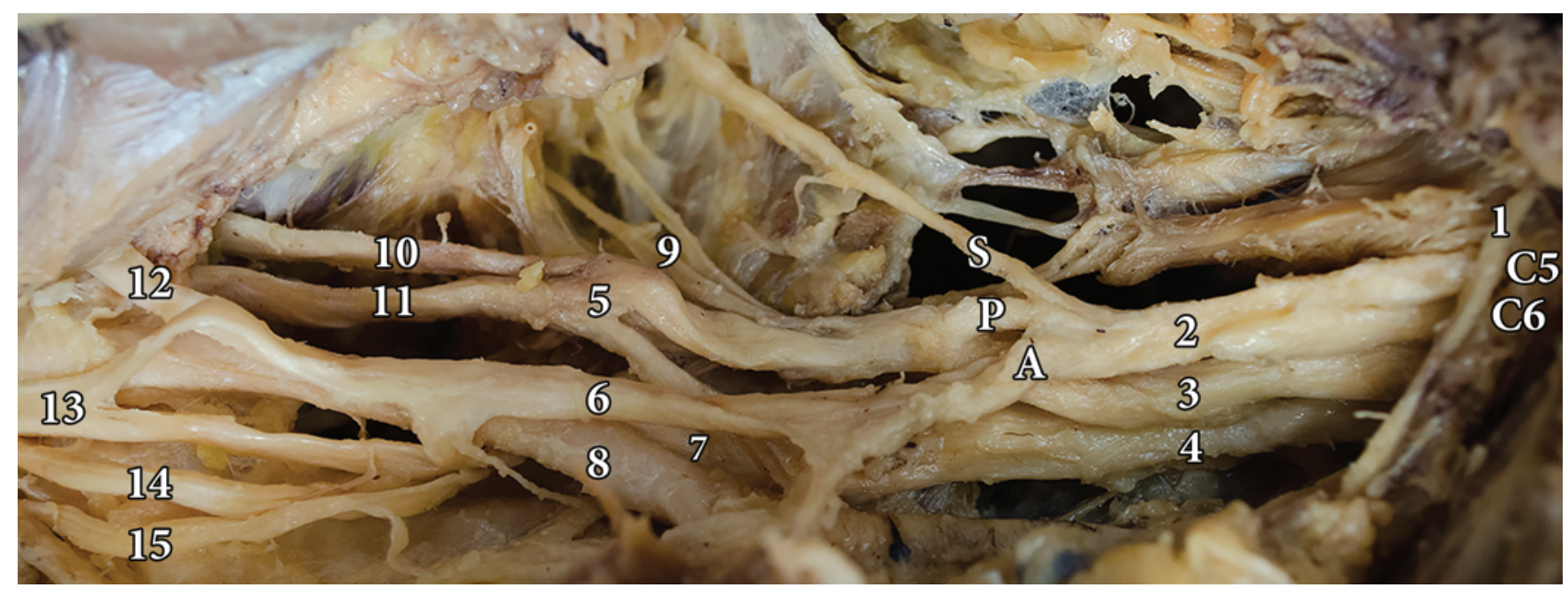

FIG. 1. Photograph showing the anterior exposure of the right brachial plexus. $1=$ phrenic nerve; $2=\mathrm{UT} ; 3=\mathrm{MT} ; 4=\mathrm{LT} ; 5=$ posterior cord; 6 = lateral cord; 7 = medial cord; $8=$ axillary artery; $9=$ upper and lower subscapular nerves; $10=$ axillary nerve; note in this specimen the posterior division of the LT meets the posterior divisions of the UT and MT after the branching of the axillary nerve; 11 = radial nerve; 12 = musculocutaneous nerve; $13=$ median nerve; $14=$ ulnar nerve; $15=$ medial antebrachial cutaneous nerve. Note the SPA arrangement from craniodorsal to caudoventral. 


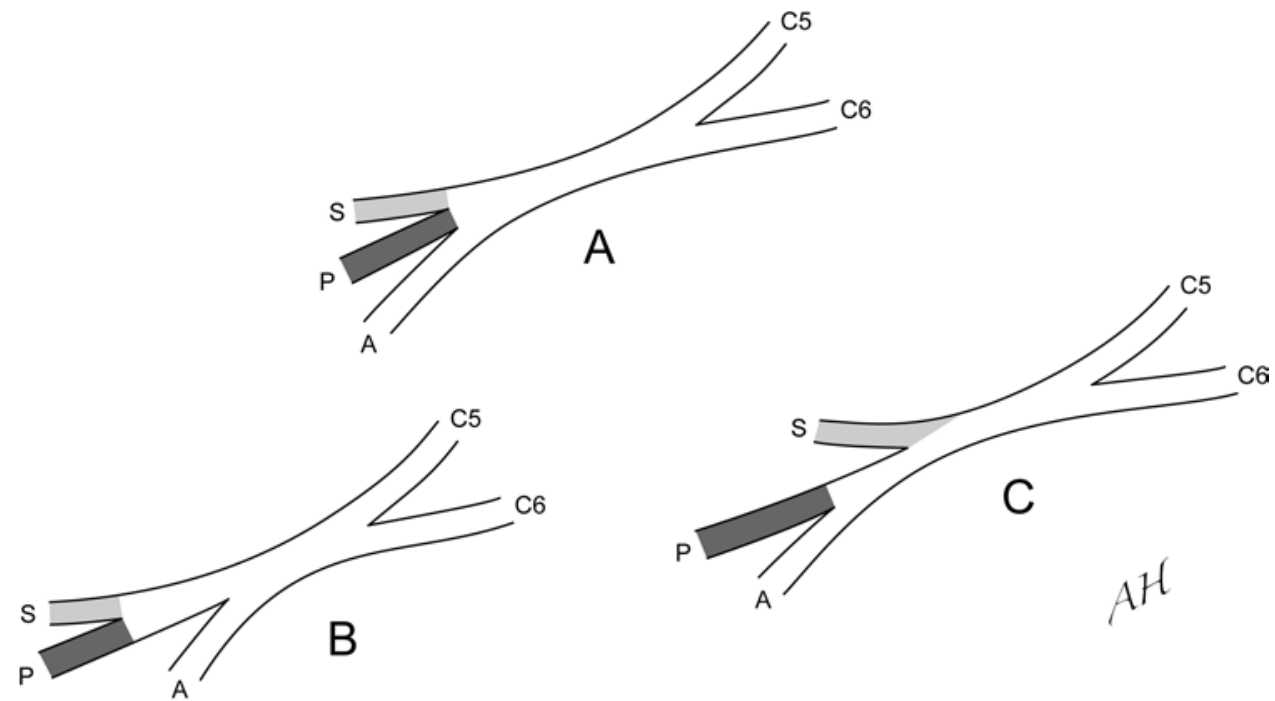

FIG. 2. Diagrammatic representations showing the different patterns of branching of the UT. The most common pattern is a trifurcation $(70 \%)(A)$, with some variability, where A may arise more proximally $(25 \%)(B)$ or $S$ arises more proximally $(5 \%)$ (C). Copyright Amgad Hanna. Published with permission.

the wrong targets. We conducted this study on 20 BPs to confirm the accurate concept, both from anterior and posterior approaches. In all specimens, with no exception, the arrangement from cranial and dorsal to caudal and ventral was: S, P, and A, sequentially. Hence I propose the acronym SPA. I am also proposing a new illustration of the BP that adopts this correct concept (Fig. 6). In a beautifully executed interfascicular dissection of the UT, Siqueira et al. studied the origin of S from the UT and illustrate nicely the trifurcation concept; however, they clearly mislabeled the anterior fascicles going to the posterior division while the posterior fascicles go to the anterior division (Fig. 1 in that report).$^{13}$ This concept is incorrect. Arad et al. studied the origin of $\mathrm{S}$ and lateral pectoral nerves from the BP. They nicely illustrate the correct concept of the SPA arrangement of the branches of the UT (Fig. 1 in that report), ${ }^{1}$ describing $\mathrm{S}$ as a branch of $\mathrm{P}$. Midha describes the trifurcation of the UT as a "trident-like structure,"10 and David et al., in a paper about transfer of pectoral nerves to suprascapular and axillary nerves, show the correct arrangement in their Fig. 3. ${ }^{3}$ In Bertelli and Ghizoni's illustration of the reconstruction of the UT branches, the

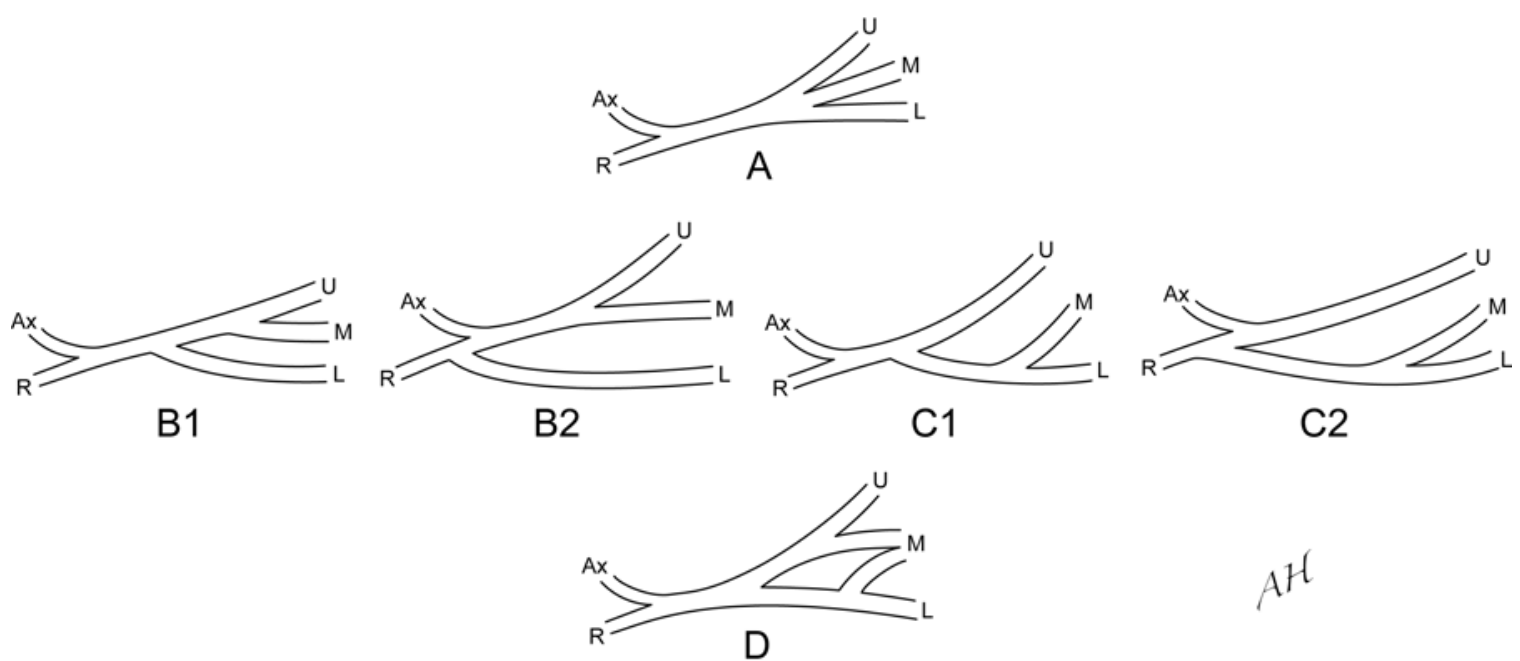

FIG. 3. Diagrammatic representations showing the different arrangements that constitute the posterior cord. Rarely, the posterior division of the UT (U), posterior division of the MT (M), and posterior division of the LT (L) met at the same spot to make the posterior cord $(6.25 \%)(\mathrm{A})$. More frequently either the posterior division of the UT and posterior division of the MT met first $(50 \%)$ (B1 and B2) or the posterior division of the MT and posterior division of the LT met first (37.5\%) (C1 and C2). They then joined the posterior division of the remaining trunk, sometimes after the take-off of the axillary nerve (B2 and $\mathbf{C} 2)$. In 1 specimen (6.25\%), the posterior division of the MT split into 2 posterior divisions, that first met the posterior division of the UT and the posterior division of the LT, respectively, and then met together to form the posterior cord (D). Ax = axillary nerve; $R=$ radial nerve. Copyright Amgad Hanna. Published with permission. 


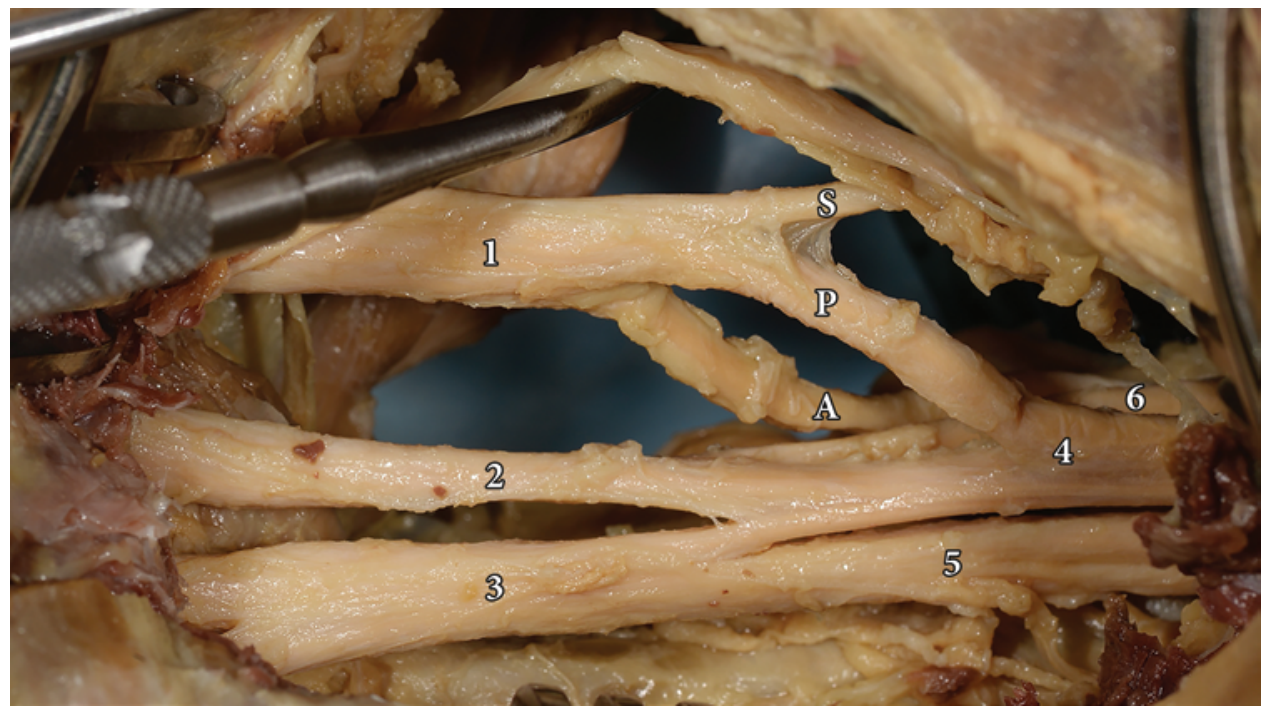

FIG. 4. Photograph showing a posterior view of the right brachial plexus. $1=U T ; 2=M T ; 3=L T ; 4=$ posterior cord (note the pattern of formation of the posterior cord corresponds to $\mathrm{C} 1$ in Fig. 3 ); $5=$ medial cord; $6=$ lateral cord. Note the branching pattern of the UT corresponds to B in Fig. 2. Note also the SPA arrangement from craniodorsal to caudoventral. The long thoracic nerve has been retracted cranially.

correct concept is adopted in the diagrams; however, the intraoperative photographs are mislabeled (Figs. 3 and 5 in that report). ${ }^{2}$ The arrangement of the branches of the UT is incorrectly illustrated in other papers as well, ${ }^{6,11}$ and few papers present accurate illustrations. ${ }^{8,12}$ Given that a branch may appear several millimeters more proximal or distal, depending on how much of the dissection is car-

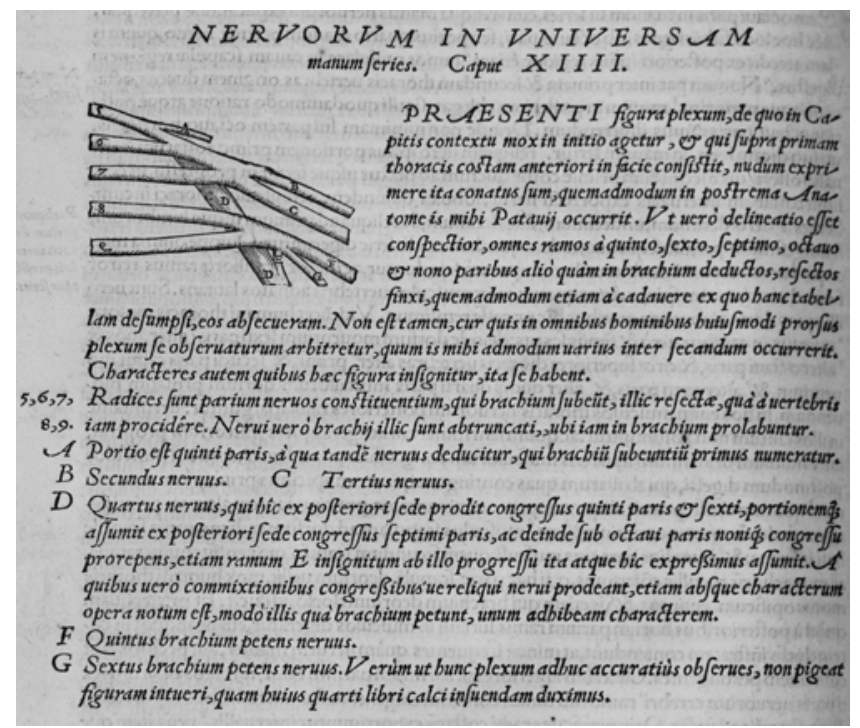

FIG. 5. A reproduction of the BP as illustrated by Vesalius in 1555. $A=$ suprascapular nerve; $B=$ musculocutaneous nerve (second nerve); $C=$ median nerve (third nerve); $\mathrm{D}=$ radial nerve (fourth nerve); $\mathrm{E}=$ posterior division of the LT; $F=$ ulnar nerve (fifth nerve); $G$ = medial antebrachial cutaneous and medial brachial cutaneous nerves (sixth nerve). Courtesy of Ebling Library, Rare Books and Special Collections, University of Wisconsin-Madison. Note the premature take-off of the suprascapular nerve from C-5 instead of UT. Also note the cranial position of the anterior division of the UT in relation to its posterior division-a misconception that has been perpetuated for centuries.

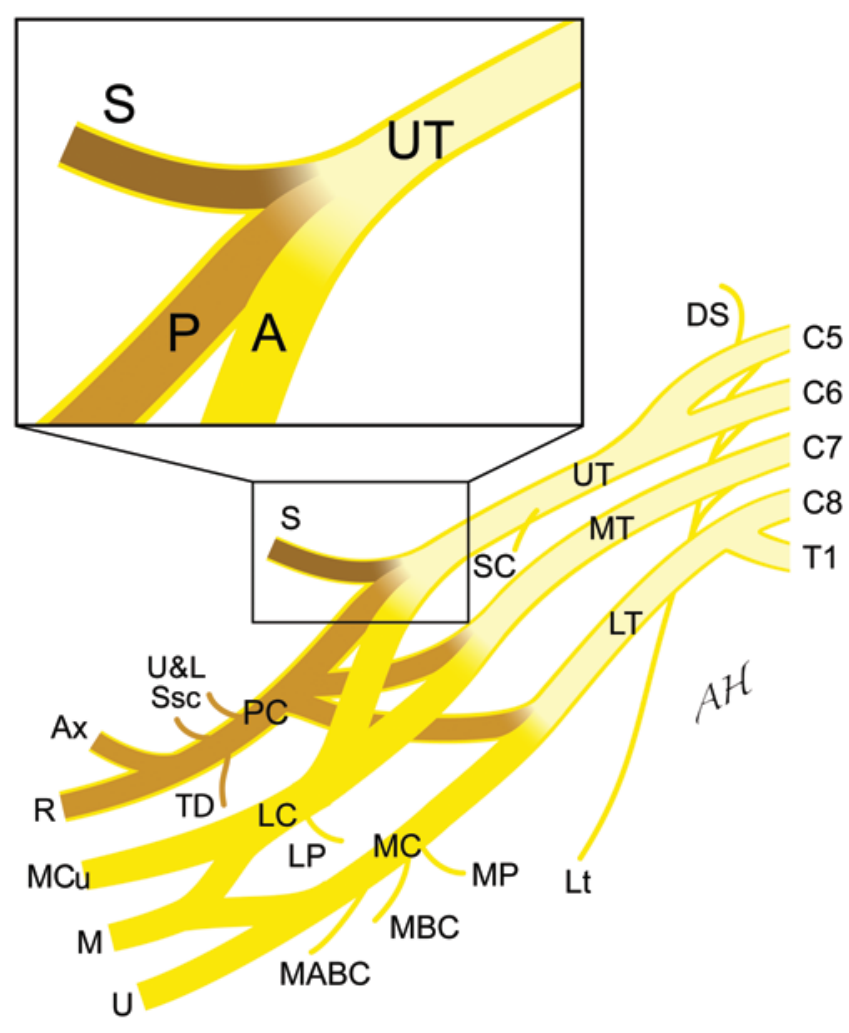

FIG. 6. Newly proposed diagram of the brachial plexus, taking into account the SPA arrangement of the trifurcation of the UT. Ax = axillary; $D S=$ dorsal scapular; $L C=$ lateral cord; $L P=$ lateral pectoral; $L t=$ long thoracic; $\mathrm{M}=$ median; $\mathrm{MABC}=$ medial antebrachial cutaneous; $\mathrm{MBC}$ = medial brachial cutaneous; $\mathrm{MC}=$ medial cord; $\mathrm{MCu}=$ musculocutaneous; $\mathrm{MP}=$ medial pectoral; $\mathrm{PC}=$ posterior cord; $\mathrm{R}=$ radial; $\mathrm{SC}=$ subclavius; TD = thoracodorsal; $U=$ ulnar; U\&L Ssc = upper and lower subscapular. Copyright Amgad Hanna. Published with permission. 
ried out interfascicularly, specific anatomical measurements were intentionally omitted from the current study. The principal and significant finding of the current study is that $100 \%$ of the specimens showed the sequential SPA arrangement of the branches of the upper trunk of the BP, a finding not clearly described in the previously published literature.

\section{Conclusions}

It is time to abandon the inaccurate and outdated drawing of the brachial plexus, and revise our understanding of the relationship between the surprascapular nerve, the posterior division, and the anterior division of the upper trunk. This anatomical relationship is shown to be consistent from cranial and dorsal to caudal and ventral: S, P, and A, sequentially, hence the acronym SPA.

\section{References}

1. Arad E, Li Z, Sitzman TJ, Agur AM, Clarke HM: Anatomic sites of origin of the suprascapular and lateral pectoral nerves within the brachial plexus. Plast Reconstr Surg 133:20e-27e, 2014

2. Bertelli JA, Ghizoni MF: Results of grafting the anterior and posterior divisions of the upper trunk in complete palsies of the brachial plexus. J Hand Surg Am 33:1529-1540, 2008

3. David S, Balaguer T, Baque P, Lebreton E: Transfer of pectoral nerves to suprascapular and axillary nerves: an anatomic feasibility study. J Hand Surg Am 35:92-96, 2010

4. Grant JCB: Atlas of Anatomy, ed 6. Baltimore: Williams \& Wilkins, 1972, p 476

5. Kline DG, Hudson AR, Kim DH: Atlas of Peripheral Nerve Surgery. Philadelphia: Saunders, 2001, pp 11, 208

6. Leinberry CF, Wehbé MA: Brachial plexus anatomy. Hand Clin 20:1-5, 2004

7. MacKinnon SE, Dellon AL: Surgery of the Peripheral Nerve. New York: Thieme, 1988, p 425

8. Malessy MJ, Pondaag W: Neonatal brachial plexus palsy with neurotmesis of C5 and avulsion of C6: supraclavicular reconstruction strategies and outcome. J Bone Joint Surg Am 96:e174, 2014

9. Maniker AH: Operative Exposures in Peripheral Nerve Surgery. New York: Thieme, 2005, p 15

10. Midha R: Brachial plexus anatomy and pre-operative physiology, in Kim DH, Midha R, Murovic JA, et al (eds): Kline and Hudson's Nerve Injuries, ed 2. Philadelphia: Saunders Elsevier, 2008, pp 282 and 290

11. Porzionato A, Macchi V, Stecco C, Loukas M, Tubbs RS, De Caro R: Surgical anatomy of the pectoral nerves and the pectoral musculature. Clin Anat 25:559-575, 2012

12. Shetty P, Nayak SB, Kumar N, Thangarajan R, D'Souza MR: Origin of medial and lateral pectoral nerves from the supraclavicular part of brachial plexus and its clinical importance-a case report. J Clin Diagn Res 8:133-134, 2014

13. Siqueira MG, Foroni LH, Martins RS, Chadi G, Malessy MJ: Fascicular topography of the suprascapular nerve in the C5 root and upper trunk of the brachial plexus: a microanatomic study from a nerve surgeon's perspective. Neurosurgery 67 (2 Suppl Operative):402-406, 2010

14. Siqueira MG, Martins RS: Lesões do plexo braquial. Rio de Janeiro: Di Livros, 2011, p 9

15. Slutsky DJ, Hentz VR (eds): Peripheral Nerve Surgery: Practical Applications in the Upper Extremity. Philadelphia: Churchill Livingstone Elsevier, 2006, p 97

16. Standring S: Gray's Anatomy: The Anatomical Basis of Clinical Practice, ed 40. London: Churchill Livingstone Elsevier, 2008, pp 781, 819

17. Tung THH, Moore AM: Brachial plexus injuries, in Mackinnon SE (ed): Nerve Surgery. New York: Thieme, 2015, p 393

18. Vesalius A: De Humani Corporis Fabrica Libri Septem, ed 2. Basel, Switzerland: Ioannem Oporinum, 1555

\section{Disclosures}

The author reports no conflict of interest concerning the materials or methods used in this study or the findings specified in this paper.

\section{Supplemental Information \\ Previous Presentations}

Portions of this work were presented to the Congress of Neurological Surgeons 2014 (October 18-22, Boston, MA), the American Society of Peripheral Nerves 2015 (January 23-25, Paradise Island, Bahamas), and the Sunderland Society meeting 2015 (June 7-9, Ann Arbor, MI).

\section{Correspondence}

Amgad Hanna, Department of Neurological Surgery, University of Wisconsin, 600 Highland Ave., Madison, WI 53792. email: ah2904@yahoo.com. 